\title{
Hipodermóclise: alternativa para infusão de medicamentos em pacientes idosos e pacientes em cuidados paliativos
}

\section{Hypodermoclysis: alternative for infusion of drugs in elderly patients and patients in palliative care}

\section{Hipodermóclisis: alternativa para la infusión de medicamentos en pacientes mayores y pacientes en cuidados paliativos}

\author{
Camila Figueiró Vasconcellos $\mathbb{1}^{1} \bowtie$, Denise Milão ${ }^{2}{ }^{2}$ \\ Hospital São Lucas, Pontifícia Universidade Católica do Rio Grande do Sul. Porto Alegre, RS, Brasil. \\ 2 Escola de Ciências da Saúde, Pontifícia Universidade Católica do Rio Grande do Sul. Porto Alegre, RS, Brasil.
}

\section{ARTICLE INFO}

\section{Article history}

Received: 19/11/2018

Accepted: 18/02/2019

Published: $21 / 05 / 2019$

$\triangle$ Correspondent Author Camila Figueiró Vasconcellos Av. Ipiranga 6681 , prédio 81 90619-900, Porto Alegre, RS, Brasi camila.vasconcellos@acad.pucrs.br

(C) 2019 All rights reserved

\section{Editors}

Alfredo Cataldo Neto

Paula Engroff

\begin{abstract}
RESUMO
OBJETIVO: Realizar uma revisão teórica a respeito do uso e aplicabilidade da terapia subcutânea e construir um guia para diluições e compatibilidades entre medicamentos, permitindo a utilização segura e eficaz por pacientes idosos ou em cuidados paliativos. FONTE DE DADOS: Como recurso de pesquisa foi aplicada a busca por artigos científicos nas bases de dados Lilacs, Medline, Pubmed e Scielo para realização de uma revisão narrativa sobre o tema, utilizando os termos de busca "Hipodermóclise", "Terapia subcutânea", "Hypodermoclysis" e "Subcutaneous".

SÍNTESE DOS DADOS: Considerando o aumento progressivo da população de idosos e do número de pessoas portadoras de doenças crônicas ou terminais, é importante que sejam desenvolvidas técnicas terapêuticas adequadas para garantir o conforto e a autonomia desses pacientes. A hipodermóclise ou terapia subcutânea, possui eficácia terapêutica comprovada e pode auxiliar pacientes que não apresentem possibilidade de utilização da via oral para administração de medicamentos e hidratação. Apresenta-se como uma forma segura, simples e com baixo risco de efeitos adversos, também atuando como recurso para alívio de sintomas como náuseas e vômitos, além de dor de difícil controle e desidratação. Entretanto, possui desvantagens como a impossibilidade de ajustes rápidos de doses e volume, velocidade de infusão limitados e a pequena quantidade medicamentos e eletrólitos que possam ser infundidos.

CONCLUSÃO: a hipodermóclise é uma via de administração segura desde que sejam consideradas as características clínicas de cada paciente. Para utilização adequada desta técnica, faz-se necessária a construção de protocolos compatíveis à realidade de cada instituição, considerando as características de cada medicamento e o cuidado necessário para promover a qualidade de vida a esses pacientes, de forma que possam atingir seus objetivos terapêuticos.
\end{abstract}

PalaVras-Chave: Cuidados paliativos; Hidratação; Hipodermóclise; Idosos; Pacientes.

\section{ABSTRACT}

AIM: execute a theoretical review about the use and applicability of subcutaneous therapy and to construct a guide for dilutions and compatibilities between drugs, allowing a safe and effective use by elderly or in need of palliative care patients. 
SOURCE OF DATA: The research resource used was the search for scientific data in databases such as Lilacs, Medline, Pubmed, and Scielo, to perform a narrative review on the subject, using the terms "Hipodermóclise", "Terapia subcutânea", "Hypodermoclysis" and "Subcutaneous".

SUMMARY OF FINDINGS: Considering the progressive increase in the elderly population and also in the number of people with chronic or terminal diseases, is important the development of techniques which ensure the comfort and the autonomy of these patients. The Hypodermoclysis or subcutaneous therapy has its therapeutical efficiency proved and it also can be helpful with patients who don't have the oral way as a possibility for medication administration and hydration. It presents a safe and low-risk way to adverse effects, also acting as relief resource to symptoms such as náusea, vomiting, hard control pain, and dehydration. However, there are some disadvantages such as the impossibility of fast doses and volume adjustment, the limited and infusion speed and the small number of medicine and electrolytes that can be infused.

CONCLUSION: Hypodermoclysis is a safe way of administration, since the clinical characteristics of each patient are considered and, in order to fit the technique, it is necessary to create a database according to the reality of each institution, considering the characteristics of each medicine and the necessary care to promote the quality of life of these patients, so that they can reach their therapeutic goals.

KEYWORDS: Aged; Hypodermoclysis; Fluid therapy; Palliative care; Patients.

\section{RESUMEN}

OBJETIVO: Realizar una revisión teórica respecto al uso y aplicabilidad de la terapia subcutánea y construir una guía para diluciones y compatibilidades entre medicamentos, permitiendo la utilización segura y eficaz por pacientes mayores o en cuidados paliativos.

FUENTE DE DATOS: Como recurso de investigación se hizo la búsqueda de artículos científicos en las bases de datos Lilacs, Medline, Pubmed y Scielo para la realización de una revisión narrativa sobre el tema, utilizando los términos de búsqueda "Hipodermóclise", "Terapia subcutânea", "Hipodermoclisis" y "Subcutaneous".

SÍNTESIS DE LOS DATOS: Considerando el aumento progresivo de la población de mayores y del número de personas portadoras de enfermedades crónicas o terminales, es importante que se desarrollen técnicas terapéuticas adecuadas para asegurar el confort y la autonomía de esos pacientes. La hipodermóclisis o terapia subcutánea, posee eficacia terapéutica comprobada y puede auxiliar a pacientes que no presenten la posibilidad de utilización de la vía oral para la administración de medicamentos e hidratación. Se presenta como una forma segura, simple y con bajo riesgo de efectos adversos, también actuando como recurso para el alivio de síntomas como náuseas y vómitos, además de dolores de difícil control y deshidratación. Sin embargo, posee desventajas como la imposibilidad de ajustes rápidos de dosis y volumen, velocidad de infusión limitados y la pequeña cantidad de medicamentos y electrólitos que puedan ser infundidos.

CONCLUSIÓN: La hipodermóclisis es una vía de administración segura siempre y cuando se consideren las características clínicas de cada paciente. Para la utilización adecuada de esta técnica, se vuelve necesaria la construcción de protocolos compatibles a la realidad de cada institución, considerando las características de cada medicamento y el cuidado necesario para promover la calidad de vida de esos pacientes, de forma que puedan alcanzar sus objetivos terapéuticos.

Palabras Clave: Cuidados paliativos; Fluidoterapia; Hipodermoclisis; Anciano; Pacientes.

\section{INTRODUÇÃO}

Atualmente podemos observar uma mudança no perfil demográfico do país, com o crescimento da população de idosos. Quadro esse proveniente de mudanças no estilo de vida, melhorias em recursos médico e tecnológicos e o decréscimo nas taxas de fecundidade e mortalidade. Tal mudança no perfil, exige uma resposta adequada das políticas públicas ${ }^{1,2}$. A Organização Pan-Americana de Saúde e a Organização Mundial da Saúde (OMS) estimam que em 2025 existirá 1,2 bilhões de pessoas com mais de 60 anos e 2 bilhões até 2050, sendo 80\% em países desenvolvidos. ${ }^{3,4}$ Pensando nesta futura realidade, foi criada a Política Nacional de Saúde da Pessoa Idosa (PNSPI) com o objetivo de garantir a assistência e atender às necessidades do idoso de forma que ele seja mantido incluso na sociedade e a sua autonomia preservada. ${ }^{5}$

Doenças crônicas como reumatismo, diabetes, doença de Alzheimer e Parkinson frequentemente acometem a população idosa, que por sua vez, necessitará de cuidados permanentes visando o seu bem-estar e qualidade de vida, indicando-se o cuidado paliativo para atingir este objetivo. De acordo com a OMS, cuidado paliativo é uma abordagem que promove qualidade de vida a pacientes e familiares ao enfrentarem doenças que ameacem a continuidade da vida, prevenindo e aliviando sofrimento, podendo ser oferecido a qualquer pessoa com doença crônica irreversível e em estágio avançado independentemente da idade. ${ }^{6,7,8}$

A via oral é a primeira escolha para pacientes idosos, porém, quando esta não é uma opção, recomenda-se a via subcutânea, por ser menos dolorosa, de fácil manejo e com menores efeitos adversos para aplicação contínua ou intermitente de fármacos e fluidos, até mesmo em domicílio. ${ }^{9}$ A administração de grandes volumes de fluidos pela via subcutânea é denominada hipodermóclise, onde a absorção de tal volume ocorre pela ação de forças hidrostáticas e osmóticas que permitem que a solução atinja o espaço intravascular. ${ }^{10,11}$

A hipodermóclise é uma técnica antiga, comumente utilizada dentro dos cuidados paliativos na 
atualidade, porém, com efetividade comprovada em 1859 pelo médico Charles Hunter com a demonstração da ação sistêmica de analgésicos pela via subcutânea, popularizando esse recurso e induzindo a exploração de novas opções de tratamento. ${ }^{10}$

Tal técnica apresenta-se como a utilizada principalmente para infusão de analgésicos e adjuvantes no controle da dor e hidratação, tanto em pacientes internados como os que se encontram em acompanhamento ambulatorial, por permitir o controle de sintomas de forma eficaz. Atualmente a dificuldade no uso da técnica se dá principalmente pela falta de documentações padronizadas, que guiem a aplicação da mesma, orientando com relação a quais medicações podem ser utilizadas, diluições e compatibilidades entre elas, podendo ocasionar em insegurança no uso por parte dos profissionais da saúde..$^{12,13,14}$

A via subcutânea vem sendo utilizada em diversas instituições de saúde nacionais e internacionais como valioso recurso para administração tanto de medicamentos quanto de soluções. Aplicada em pacientes que não possuem a possibilidade da utilização da via oral por problemas com a deglutição ou da via endovenosa, onde a fragilidade das veias e a perda de elasticidade não permitem a punção de um acesso venoso, além de permitir maior conforto, praticidade e menores riscos quando comparada a um cateter venoso central. ${ }^{15,16}$

O presente trabalho possuiu o objetivo de realizar uma revisão teórica a respeito do uso da hipodermóclise e construir um material de consulta com informações atuais sobre a administração de medicamentos e soluções para auxiliar na rotina dos diferentes profissionais que trabalham com este recurso, de forma que colabore para o uso seguro e eficiente desta via que se torna cada vez mais um importante recurso para pacientes idosos ou em cuidados paliativos.

\section{METODOLOGIA}

Trata-se de uma revisão da literatura a respeito da aplicação de hipodermóclise como opção terapêutica para pacientes idosos ou em cuidados paliativos. Foi utilizado como recurso de pesquisa a busca por artigos científicos nas bases de dados eletrônicas Lilacs, Medline, PubMed e Scielo e cinco manuais publicados sobre a temática para construção de uma revisão bibliográfica narrativa.

Os seguintes termos de busca foram definidos por convenção entre os autores, "Hipodermóclise", "Terapia subcutânea", "Hypodermoclysis" e "Subcutaneous"; como critérios de inclusão selecionamos artigos publicados em português ou inglês no período de 2007 a 2017 que abordassem integralmente o tema.
Foram encontrados 3.245 resultados durante a pesquisa em bases de dados. Desses, 12 publicações foram selecionadas para análise, aplicando-se como critério de exclusão a incompatibilidade com o tema de estudo. (Lilacs: 2.287 artigos encontrados e 2 artigos selecionados, Medline: 151 artigos encontrados e 2 artigos selecionados, Scielo: 598 artigos encontrados e 4 artigos selecionados, Pubmed: 209 artigos encontrados e 4 artigos selecionados). Empregaramse também cinco manuais sobre a terapia subcutânea disponibilizados online de forma gratuita.

\section{REVISÃO DE LITERATURA}

A partir dos trabalhos selecionados para a pesquisa, foram extraídos os seguintes tópicos relacionados às características da via, da técnica de hipodermóclise e da administração de medicamentos e soluções.

\section{A hipodermóclise como recurso}

No Brasil, os primeiros serviços de cuidados paliativos surgiram por volta de 1970-80 como iniciativas isoladas. Os serviços mais organizados, que iniciaram em torno dos anos 90, são considerados uma ciência jovem. ${ }^{8,17}$ Indica-se iniciar o cuidado no estágio inicial da doença, mesmo que sejam oferecidas em conjunto terapias modificadoras da doença e/ou prolongadoras da vida. ${ }^{18,19}$

A expectativa de vida de pessoas com doenças crônicas têm aumentado. Pacientes idosos ou em cuidados paliativos frequentemente precisam de vias alternativas para a administração de medicamentos e manutenção da hidratação pela impossibilidade de disponibilidade das vias oral e endovenosa. A hipodermóclise possui eficácia terapêutica, é um recurso que possibilita um maior conforto e preserva a autonomia do paciente por ser um método simples e seguro, com bom custo benefício e de rápido manuseio, que pode ser implementado em ambiente hospitalar ou domiciliar. ${ }^{11,16,20,21,22}$

Pontalti et al. (2012), ${ }^{14}$ indicou em seu estudo que o principal motivo referido para a não utilização da via é a falta de experiência, concordando com o trabalho de Gomes et al. (2017), ${ }^{23}$ que relata a dificuldade da equipe de enfermagem em receber orientações sobre esta prática. As indicações de uso variam, sendo o objetivo principal proporcionar melhorias na qualidade de vida, como por exemplo: demência avançada com disfagia, náuseas e vômitos por períodos prolongados, intolerância gástrica, obstrução intestinal, diarréia, confusão mental, dispnéia intensa e pacientes oncológicos sem outras possibilidades de acesso venoso por flebite ou outros inconvenientes. ${ }^{11,15,20}$ 
São vantagens da administração por esta via: $15,20,21,23,24,25$

- Fácil inserção e manutenção do cateter;

- Realização em diferentes ambientes de cuidado (hospitalar, ambulatorial, domiciliar) possibilitando alta precoce;

- Conforto;

- Praticidade para realizar a administração;

- Baixo risco de eventos adversos e complicações sistêmicas;

- Redução da flutuação das concentrações plasmáticas de opióides;

- Baixo custo;

- Redução de hospitalizações;

- A infusão pode ser interrompida sem riscos de trombose e disfuncionalidade do acesso;

- Não exige materiais complexos.

- Ainda assim, é um recurso que também possui desvantagens: $15,20,21,23,24,25$

- Volume e velocidade de infusão limitados;

- Absorção variável (influenciada por perfusão e vascularização);

- Não permite ajuste rápido de doses;

- Suplementos nutricionais e soluções hipertônicas não são indicados;

Limitação de medicamentos e eletrólitos que podem ser infundidos.

No trabalho de Bruno (2015), ${ }^{21}$ foram encontrados dados referentes ao uso de hipodermóclise para recuperação da hidratação em idosos pacientes de uma instituição de longa permanência. Neles, $88 \%$ apresentaram melhora do quadro geral e $84 \%$ demonstraram melhoras inclusive na cognição. Também identificou-se que as drogas mais frequentemente prescritas são morfina, haloperidol, furosemida e metoclopramida, concordando com o estudo de Justino et al. (2013), ${ }^{26}$ que aponta como principais usos da terapia o alívio de sintomas como náuseas e vômitos, desidratação e dor de difícil controle.

São consideradas contraindicações absolutas para a realização da técnica: recusa do paciente, falência circulatória, anasarca, sobrecarga de fluidos (insuficiência congestiva, edema acentuado) trombocitopenia grave, hematomas, necessidade de reposição rápida de volume (desidratação grave ou choque)..$^{15,25,27}$

Já as contraindicações relativas incluem: ascite, áreas com circulação linfática comprometida (após cirurgia ou radioterapia), áreas de infecção/inflamação/ ulceração cutânea, proximidades de articulação, proeminências ósseas e também deve-se evitar a aplicação direta em locais com presença de tumores ou em pacientes em diálise peritoneal. ${ }^{10,11,15,27}$

\section{A via subcutânea e a técnica de hipodermóclise}

O maior órgão do corpo humano, a pele, é responsável por manter a integridade do corpo, absorver e excretar líquidos, regular a temperatura e até mesmo metabolizar vitaminas. A absorção de medicamentos pela via subcutânea é uniforme e lenta, dependente dos capilares sanguíneos e linfáticos, o transporte ocorre por difusão capilar e perfusão tecidual, sendo assim, essa via elimina o primeiro passo do metabolismo hepático

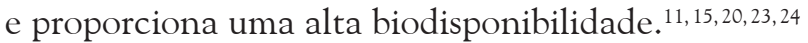

Os sítios de punção (Figura 1) devem ser escolhidos considerando as características clínicas de cada paciente, o conforto, a mobilidade e independência do indivíduo. É importante também levar em consideração que, em pacientes desnutridos, pode ocorrer a diminuição da espessura do tecido subcutâneo. As áreas indicadas para punção possuem capacidades volumétricas de absorção diferentes permitindo a administração de volumes de até $1500 \mathrm{~mL}$ em $24 \mathrm{~h}$ por sítio de punção, sendo viável a realização de dois sítios distintos que totalizem $3000 \mathrm{~mL}$ por dia. ${ }^{11,15,16,20}$

- Abdominal: capacidade de até $1000 \mathrm{~mL} / 24 \mathrm{~h}$

- Torácica: capacidade de até $250 \mathrm{~mL} / 24 \mathrm{~h}$

- Deltóide: capacidade de até $250 \mathrm{~mL} / 24 \mathrm{~h}$

- Escapular: capacidade de até $1000 \mathrm{~mL} / 24 \mathrm{~h}$

- Anterolateral da coxa: $1500 \mathrm{~mL} / 24 \mathrm{~h}$
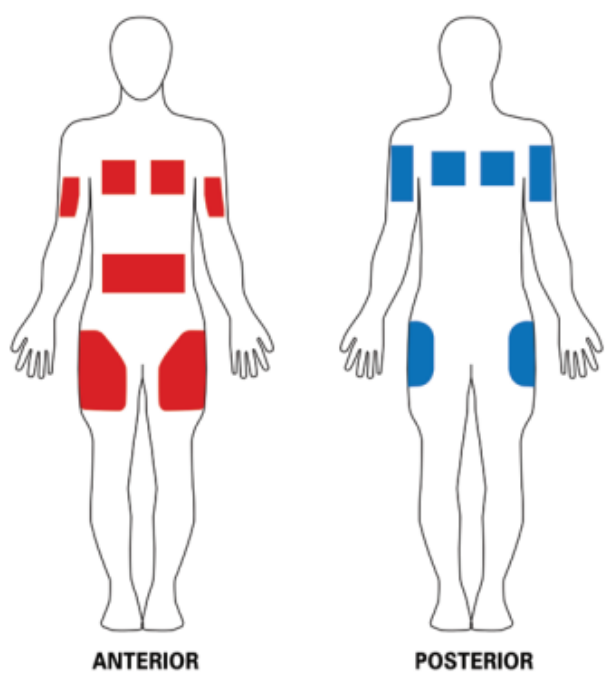

Figura 1. Sítios de punção para hipodermóclise segundo Bruno $(2015)^{21}$

Deve-se considerar a direção da drenagem linfática ao inserir o cateter, é indicado que aponte no mesmo sentido com a finalidade de reduzir as chances de formação de edemas. As áreas abdominal e torácica são as mais comumente utilizadas por suportarem maiores 
volumes. Em pacientes com caquexia, recomendase evitar a região anterior do tórax pelo risco de pneumotórax; a região interscapular é menos utilizada, porém, útil em pacientes agitados com tendência a arrancar acessos, como em idosos com delirium ou demência. ${ }^{15,20}$

Para realizar a punção pode ser utilizado um cateter agulhado, como um escalpe (butterfly) ou não-agulhado como Abocath ${ }^{\mathrm{TM}}$ (fabricante: Amsino Medical, Shanghai, China), Jelco ${ }^{\mathrm{TM}}$ (fabricante: Medex Medical, Reino Unido) e saf-T-Intima ${ }^{\mathrm{TM}}$ (fabricante: Becton, Dickinson and Company, Estados Unidos). A literatura indica uma variação de 18 a $25 \mathrm{G}$ no calibre, de acordo com a quantidade de tecido do paciente e o volume a ser infundido. $\mathrm{O}$ uso de cateteres flexíveis é preferível ao uso do butterfly e deve ser inserido com um ângulo de $30^{\circ}$ a $45^{\circ}$ em relação a pele, direcionado para o centro do corpo. No local, também deve ser aplicado preferencialmente uma película de filme transparente com a data da punção e as iniciais do profissional. ${ }^{10,11,16}$

Em seu artigo, Gomes et al. (2017), ${ }^{23}$ traz uma comparação entre o uso do abocath e do escalpe realizada no Canadá, demonstrando que, mesmo com o tempo de permanência maior do abocath, foi preferível a padronização do uso do escalpe pelo menor custo e facilidade de punção.

Recomenda-se a troca dos locais de punção a cada sete dias, a cobertura estéril transparente pode permanecer pelo mesmo período (varia de acordo com o fabricante). Já as coberturas com esparadrapo e fita micropore deverão ser trocadas diariamente. Tal manipulação pode levar a diminuição de vida útil da punção pelo aumento do risco de contaminação. ${ }^{10,15}$

Existem relatos de casos com raras complicações como perfuração intestinal, sangramento excessivo, necrose tecidual e choque. Porém, tais eventos podem estar relacionados com falhas na organização da técnica ou condições clínicas dos pacientes. ${ }^{12,13}$

Sinais e sintomas de complicações de punção e ações:

- Edema, calor, rubor ou dor prolongados: retirar acesso, fazer nova punção a pelo menos $5 \mathrm{~cm}$ de distância;

- Celulite: compressa gelada por $15 \mathrm{~min}$, curva térmica, comunicar equipe médica (considerar uso de antibiótico tópico ou sistêmico), acompanhamento diário pela enfermagem;

- Secreção purulenta: retirar acesso, drenagem manual, limpeza com SF 0,9\% e aplicação de clorexidina alcoólica 5\%, curativo oclusivo com troca pelo menos a cada $24 \mathrm{~h}$, comunicar equipe médica (considerar uso de antibiótico sistêmico ou tópico), acompanhamento diário pela enfermagem;

- Endurecimento: retirar acesso, fazer nova punção a pelo menos $5 \mathrm{~cm}$ de distância;

- Hematoma: retirar acesso, aplicar polissulfato de mucopolissacarídeo com massagem local de $4 / 4 \mathrm{~h}$, fazer nova punção com cateter não agulhado;

- Necrose: retirar acesso, curativo diário (avaliar indicação de debridamento com papaína ou hidrogel), acompanhamento diário pela enfermagem. ${ }^{18}$

\section{Administração de medicamentos e fluidos}

Recomenda-se que a dose administrada pela via subcutânea seja menor que a da via oral. Na aplicação subcutânea obtém-se uma maior biodisponibilidade dos medicamentos e a administração de eletrólitos é possível desde que eles sejam diluídos em soluções. O comportamento farmacocinético é semelhante da via intramuscular, porém, com concentração sérica menor e tempo de ação prolongado, evita-se o primeiro passo do metabolismo hepático, possibilitando alta biodisponibilidade e uma concentração sérica estável. ${ }^{15,16,20,24}$

A literatura refere que os efeitos adversos da administração de fármacos pela via subcutânea são raros e facilmente evitáveis. Algumas características podem sinalizar a presença de complicações com o acesso e recomenda-se o registro em prontuário de tais reações quando percebidas. Relatos de dor ou fácies de dor (pacientes incapazes de comunicação verbal, como na demência) são os principais indícios de que a punção está fora do espaço subcutâneo. ${ }^{4,15}$

Sabe-se que sangramento e hematomas são conhecidos como complicações do uso de hipodermóclise, porém, recomenda-se cautela no uso desta técnica em pacientes que recebem heparina com intuito de prevenir sangramentos severos que podem ser prejudiciais ao quadro clínico. ${ }^{28}$

Os medicamentos administrados devem estar na forma líquida, podem ser aplicados em bolus ou em infusão contínua. Caso os volumes administrados sejam superiores a $1500 \mathrm{~mL}$ por dia, um segundo acesso se faz necessário. Em caso de medicamentos incompatíveis para infusão pelo mesmo acesso, também deve-se puncionar um segundo. ${ }^{15,20}$

Com frequência a administração de medicamentos via subcutânea é off-label, sendo assim, o volume final das diluições pode ser variável, de acordo com a experiência de uso observada na prática da instituição e com fatores clínicos do paciente, como em casos de restrição hídrica por exemplo. 15,11,20,21,29 
São compatíveis com a via medicamentos líquidos, hidrossolúveis e os com $\mathrm{pH}$ próximos da neutralidade $(7,38-7,45)$, alguns com $\mathrm{pH}$ ácido como haloperidol $(3,0-3,8)$ e metoclopramida $(3,0-5,0)$ podem ser administrados desde que seja feito de forma mais lenta, porém, soluções com $\mathrm{pH}$ extremos $(<2$ ou $>11$ ) apresentam risco aumentado para precipitação ou irritação local, sendo incompatíveis com essa via. Recomenda-se realizar a administração logo em seguida ao preparo a fim de preservar a estabilidade do medicamento. ${ }^{15,11,20,21,29}$

O trabalho de Vidal et al. (2016), ${ }^{30}$ demonstrou que, ao usar a hipodermóclise para recuperação da hidratação, foram poucos os casos em que ocorreram efeitos adversos, com 5\% dos pacientes apresentando vazamento e $12 \%$ desconforto local.

Para diluição dos medicamentos não existe um consenso claro. As rotinas de cada serviço podem divergir entre si; recomendando-se a diluição para minimizar a irritação no local da punção, são indicados o uso de água destilada e soro fisiológico 0,9\%:

- Água destilada: possui uma maior quantidade de dados disponíveis, porém, volumes maiores são hipotônicos e podem causar dor no local da infusão. ${ }^{11,15,20}$

- Soro fisiológico 0,9\%: isotônico e preferível para diluição de drogas irritativas. . $^{11,15,20}$

Recomenda-se que cada sítio de punção receba no máximo três drogas compatíveis entre si, administradas em tempos diferentes para que em caso de reações inesperadas, possa-se saber o medicamento que a causou. A incompatibilidade contribui para lesões precoces no tecido e para minimizar esse risco indicase o uso de tabelas de diluição e compatibilidade entre medicamentos (Tabelas 1, 2, 3 e 4).

Tabela 1. Diluições de medicamentos para administração pela via subcutânea. ${ }^{1,13,15,21,22,31}$

\begin{tabular}{|c|c|c|c|c|}
\hline Medicamento & Dose (usual) & Diluente & Tempo de infusão & Observações \\
\hline Ampicilina & $1 \mathrm{~g} / \mathrm{dia}$ & SF $0,9 \% 50 \mathrm{ml}$ & $60 \mathrm{~min}$ & Seguir padrão de $1 \mathrm{ml} / \mathrm{min}$ ou $62,5 \mathrm{ml} / \mathrm{h}$ \\
\hline Cefepime & $\operatorname{lg~} 12 / 12 \mathrm{~h}$ ou $8 / 8 \mathrm{~h}$ & SF $0,9 \% 100 \mathrm{~mL}$ & $60 \mathrm{~min}$ & $\begin{array}{l}\text { Seguir padrão de } 62,5 \mathrm{ml} / \mathrm{h} \text { ou } 1 \mathrm{ml} / \mathrm{min} \\
\text { - Estudos demonstram possibilidade de } \\
\text { tempo mínimo de } 40 \mathrm{~min}\end{array}$ \\
\hline Cefotaxima & $500 \mathrm{mg} / \mathrm{dia}$ & SF $0,9 \%$ & $30 \mathrm{~min}$ & \\
\hline Ceftazidima & $500 \mathrm{mg} / \mathrm{dia}$ & SF $0,9 \%$ & $30 \mathrm{~min}$ & \\
\hline Ceftriaxona & $1 \mathrm{~g} 12 / 12 \mathrm{~h}$ & SF $0,9 \% 100 \mathrm{~mL}$ & $60 \mathrm{~min}$ & $\begin{array}{l}\text { Seguir padrão de } 62,5 \mathrm{ml} / \mathrm{h} \text { ou } 1 \mathrm{ml} / \mathrm{min} \\
\text { - Estudos demonstram possibilidade de } \\
\text { tempo mínimo de } 40 \mathrm{~min}\end{array}$ \\
\hline Cetorolaco & $30-90 \mathrm{mg} / \mathrm{dia}$ & SF $0,9 \%$ & & Via exclusiva \\
\hline Ciclizina & $25-50 \mathrm{mg} 8 / 8 \mathrm{~h}$ & $\mathrm{AD}$ & & Incompatível com SF \\
\hline Dexametasona & $2-16 \mathrm{mg} / \mathrm{dia}$ & $\begin{array}{l}\text { SF } 0,9 \% \\
\text { - Diluição mínima: diluir } \\
\text { proporcionalmente }(1 \mathrm{~mL} \\
\text { do medicamento em } 1 \mathrm{~mL} \\
\text { de SF) } \\
\text { - Diluição para infusão } \\
\text { continua: diluir em no } \\
\text { mínimo } 60 \mathrm{~mL} \text { de SF por } \\
\text { ser muito irritante }\end{array}$ & $60 \mathrm{~min}$ & Via exclusiva \\
\hline Diclofenaco & $75-150 \mathrm{mg} / \mathrm{dia}$ & SF $0,9 \% 30 \mathrm{ml}$ & & Pode causar irritação local. \\
\hline Dimenidrato & $50-100 \mathrm{mg} / \mathrm{dia}$ & SF $0,9 \% 1 \mathrm{ml}$ & & \\
\hline Dipirona & $1-2 \mathrm{~g}$ até $6 / 6 \mathrm{~h}$ & SF $0,9 \% 20 \mathrm{ml}$ & $20 \mathrm{~min}$ & $\begin{array}{l}\text { Via exclusiva. - Seguir o padrão de } \\
1 \mathrm{ml} / \mathrm{min}\end{array}$ \\
\hline Ertapenem & $1 \mathrm{~g} / \mathrm{dia}$ & $\begin{array}{l}\text { SF } 0,9 \% \\
\text { - Reconstituir em } 10 \mathrm{ml} \mathrm{de} \\
\text { AD e diluir em } 50 \mathrm{ml} \text { de SF }\end{array}$ & $60 \mathrm{~min}$ & Seguir padrão de $1 \mathrm{ml} / \mathrm{min}$ ou $62,5 \mathrm{ml} / \mathrm{h}$ \\
\hline Escopolamina & $\begin{array}{l}20 \mathrm{mg} 8 / 8 \mathrm{~h} \text { até } 60 \mathrm{mg} \\
6 / 6 \mathrm{~h}\end{array}$ & SF $0,9 \% 50 \mathrm{ml}$ & $50 \mathrm{~min}$ & Seguir padrão de $1 \mathrm{ml} / \mathrm{min}$ ou $62,5 \mathrm{ml} / \mathrm{h}$ \\
\hline Fenitoína & $100 \mathrm{mg} 8 / 8 \mathrm{~h}$ & SF $0,9 \% 100 \mathrm{ml}$ & & \\
\hline Fenobarbital & $100-600 \mathrm{mg} / \mathrm{dia}$ & SF $0,9 \% 00 \mathrm{ml}$ & $60 \mathrm{~min}$ & $\begin{array}{l}\text { Via exclusiva-Sempre diluir: risco } \\
\text { de necrose tecidual; pode causar dor e } \\
\text { irritação local }\end{array}$ \\
\hline
\end{tabular}


Tabela 1 (continuação)

\begin{tabular}{|c|c|c|c|c|}
\hline Medicamento & Dose (usual) & Diluente & Tempo de infusão & Observações \\
\hline Fentanil & $\begin{array}{l}\text { Usual: } 10-1000 \mathrm{mcg} / \mathrm{dia} \\
\text { Resgate: } 10 \mathrm{mcg} \text { a cada } 1 \mathrm{~h}\end{array}$ & SF $0,9 \% 50 \mathrm{~mL}$ & & Infusão contínua ACM \\
\hline Furosemida & $20-140 \mathrm{mg} / \mathrm{dia}$ & $\begin{array}{l}\text { SF } 0,9 \% \\
\text { - Diluir } 1 \text { ampola de } 20 \mathrm{mg} \\
\text { em SF } 0,9 \% 10 \mathrm{~mL}\end{array}$ & & $\begin{array}{l}\text { Seguir padrão de } 1 \mathrm{~mL} / \mathrm{min} \text { ou infusão } \\
\text { contínua para volumes maiores }\end{array}$ \\
\hline Granisetrona & $3-9 \mathrm{mg} / \mathrm{dia}$ & SF $0,9 \% 50 \mathrm{~mL}$ & $>10 \mathrm{~min}$ & \\
\hline Haloperidol & $0,5-30 \mathrm{mg} / \mathrm{dia}$ & $\begin{array}{l}\mathrm{AD} \text { ou SF } 0,9 \% 5 \mathrm{~mL}(\mathrm{se} \\
\text { concentração até } 1 \mathrm{mg} / \mathrm{mL})\end{array}$ & & $\begin{array}{l}\text { Se concentração } \geq 1 \mathrm{mg} / \mathrm{mL} \text { usar } \mathrm{AD} \\
\text { como diluente (precipitação no SF } 0,9 \% \text { ) }\end{array}$ \\
\hline Hioscina & $0,8-3,2 \mathrm{mg} / \mathrm{dia}$ & SF $0,9 \%$ ou $\mathrm{AD}$ & & Incompatível com Ciclizina \\
\hline Ketamina & $\begin{array}{l}100-150 \mathrm{mg} / \text { dia (infusão } \\
\text { continua). Aumentar a } \\
\text { dose gradativamente } \\
(100 \mathrm{mg} / \text { dia), pode ser } \\
\text { aumentada até } 2,4 \mathrm{~g} / \text { dia }\end{array}$ & SF $0,9 \% 100 \mathrm{~mL}$ & & Rotação de punção para evitar necrose \\
\hline Levomepromazina & Até $25 \mathrm{mg} / \mathrm{dia}$ & SF $0,9 \% 30 \mathrm{~mL}$ & Fotossensivel & Irritante \\
\hline Meropenem & $500 \mathrm{mg}-1 \mathrm{~g} 8 / 8 \mathrm{~h}$ & SF $0,9 \% 100 \mathrm{ml}$ & $\begin{array}{l}60 \text { min } \\
\text { - A solução é } \\
\text { estável por } 3 \mathrm{~h} \\
\text { em temperatura } \\
\text { ambiente após } \\
\text { reconstituição } \\
\text { ou } 15 \mathrm{~h} \text { após } \\
\text { reconstituição }\end{array}$ & Seguir padrão de $1 \mathrm{ml} / \mathrm{min}$ ou $62,5 \mathrm{ml} / \mathrm{h}$ \\
\hline Metadona & $50 \%$ da dose oral habitual & SF $0,9 \% 10 \mathrm{~mL}$ & $60 \mathrm{ml} / \mathrm{h}$ & $\begin{array}{l}\text { Irritante (variar o local da punção a } \\
\text { cada } 24 \mathrm{~h} \text { ) }\end{array}$ \\
\hline Metoclopramida & $30-120 \mathrm{mg} / \mathrm{dia}$ & SF $0,9 \% 50 \mathrm{ml}$ & $30 \mathrm{~min}$ & Irritante \\
\hline Midazolam & $\begin{array}{l}1-5 \mathrm{mg} \text { (bolus) } 10- \\
120 \mathrm{mg} / \text { dia (infusão } \\
\text { contínua) }\end{array}$ & $\begin{array}{l}\text { SF } 0,9 \% 5 \mathrm{~mL} \text { (bolus) } \\
\text { SF } 0,9 \% 100 \mathrm{~mL} \text { (infusão } \\
\text { contínua) }\end{array}$ & $\begin{array}{l}\text { Infusão continua } \\
\text { ACM }\end{array}$ & Pode causar irritação local \\
\hline Morfina & $\begin{array}{l}\text { Dose inicial: } 2-3 \mathrm{mg} 4 / 4 \mathrm{~h} \\
\text { (bolus) ou } 10-20 \mathrm{mg} / \mathrm{dia} \\
\text { (infusão continua) }\end{array}$ & $\begin{array}{l}\text { Não requer diluição } \\
\text { quando em bolus } \\
\text { SF } 0,9 \% 100 \mathrm{~mL} \text { (infusão } \\
\text { continua) }\end{array}$ & $\begin{array}{l}\text { Aplicação em } \\
\text { bolus para doses } \\
\text { de resgate ou } \\
\text { infusão continua } \\
\text { ACM }\end{array}$ & $\begin{array}{l}\text { Não existe dose máxima } \\
\text { - Iniciar com a menor dose possível em } \\
\text { pacientes idosos, frágeis ou com doença } \\
\text { renal crônica }\end{array}$ \\
\hline Octreotide & $\begin{array}{l}300-900 \mathrm{mcg} / \mathrm{dia} \text { em } \\
\text { bolus ou infusão continua }\end{array}$ & $\begin{array}{l}\text { SF } 0,9 \% 5 \mathrm{~mL} \text { (bolus) ou } \\
100 \mathrm{~mL} \text { (infusão contínua) }\end{array}$ & & $\begin{array}{l}\text { Armazenamento em refrigerador, deve } \\
\text { atingir a temperatura ambiente antes da } \\
\text { administração. }\end{array}$ \\
\hline Omeprazol & $40 \mathrm{mg} \mathrm{24/24h}$ & SF $0,9 \% 100 \mathrm{~mL}$ & $4 \mathrm{~h}$ & $\begin{array}{l}\text { Via exclusiva - O medicamento deve } \\
\text { ser reconstituído com o diluente que } \\
\text { acompanha o produto, a utilização de } \\
\text { outro diluente pode alterar o pH }\end{array}$ \\
\hline Ondansetrona & $8-32 \mathrm{mg} / \mathrm{dia}$ & SF $0,9 \% 50 \mathrm{ml}$ & $50-60 \mathrm{~min}$ & Seguir padrão de $1 \mathrm{ml} / \mathrm{min}$ ou $62,5 \mathrm{ml} / \mathrm{h}$ \\
\hline Ranitidina & $\begin{array}{l}50-150 \mathrm{mg} / \text { dia (máximo } \\
300 \mathrm{mg} \text { ) }\end{array}$ & SF $0,9 \% 50 \mathrm{ml}$ & $50 \mathrm{~min}$ & Seguir padrão de $1 \mathrm{ml} / \mathrm{min}$ ou $62,5 \mathrm{ml} / \mathrm{h}$ \\
\hline Tramadol & $100-600 \mathrm{mg} / \mathrm{dia}$ & $\begin{array}{l}\text { SF } 0,9 \% 20 \mathrm{~mL} \text { (bolus) ou } \\
100 \mathrm{ml} \text { (infusão contínua) }\end{array}$ & $120 \mathrm{~min}$ & $\begin{array}{l}\text { Infusão lenta. Seguir padrão de } 1 \mathrm{ml} / \mathrm{h} \text { ou } \\
62,5 \mathrm{ml} / \mathrm{h}\end{array}$ \\
\hline
\end{tabular}

* SF 0.9\%: soro fisiológico 0,9\%; AD: água destilada.

Tabela 2. Diluições de soros para administração pela via subcutânea. , $13,15,21,22,31^{2}$

\begin{tabular}{lll}
\hline Soro & Volume & Velocidade \\
sF $0,9 \%$ & Máximo $1500 \mathrm{ml} \mathrm{em} 24 \mathrm{~h}$ por sitio & Volume de infusão máximo $62,5 \mathrm{ml} / \mathrm{h}$ \\
SGF & Máximo $1500 \mathrm{ml} \mathrm{em} 24 \mathrm{~h}$ por sitio & Volume de infusão máximo $62,5 \mathrm{ml} / \mathrm{h}$ \\
SG $5 \%$ & Máximo $1000 \mathrm{ml} \mathrm{em} 24$ por sitio & Volume de infusão máximo $62,5 \mathrm{ml} / \mathrm{h}$ \\
\hline
\end{tabular}

* SF 0.9\%: soro fisiológico 0,9\%; SGF: soro glicofisiológico, SG 5\%: soro glicosado $5 \%$. 
Tabela 3. Diluições de eletrólitos para administração pela via subcutânea. ${ }^{1,13,15,21,22,31}$

\begin{tabular}{lll}
\hline Eletrólitos & Diluição & Infusão \\
$\mathrm{NaCl} 20 \%$ & Sempre diluído em SF 0,9\% ou SG5\% - volume superior & Volume de infusão máximo 62,5 $\mathrm{ml} / \mathrm{h}$ \\
& a $100 \mathrm{ml}$ \\
KCL 19,1\% & $\begin{array}{l}\text { Sempre diluído em SF ou SG5\% - volume superior a } \\
100 \mathrm{ml}\end{array}$ & Até $40 \mathrm{mEq} /$ litro - volume de infusão máximo 62,5 ml/h \\
& \\
\hline
\end{tabular}

* SF 0.9\%: soro fisiológico 0,9\%; SG 5\%: soro glicosado 5\%.

Tabela 4. Compatibilidade de medicamentos para administração pela via subcutânea ${ }^{1,13,15,21,22,31}$

\begin{tabular}{|c|c|c|c|c|c|c|c|c|c|c|c|c|c|c|c|c|c|c|c|c|c|c|c|c|c|c|}
\hline MEDICAMENTOS & 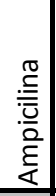 & 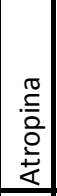 & 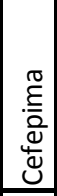 & 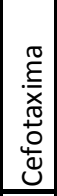 & 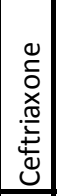 & 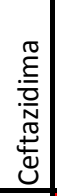 & 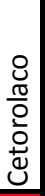 & 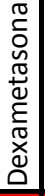 & $\begin{array}{l}\frac{0}{0} \\
\frac{0}{2} \\
.0 \\
.0 \\
0\end{array}$ & 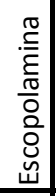 & 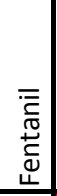 & 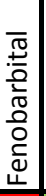 & 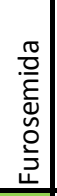 & $\begin{array}{l}\overline{0} \\
\frac{0}{0} \\
\frac{0}{0} \\
\frac{0}{0} \\
\frac{\pi}{10} \\
\end{array}$ & 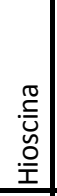 & 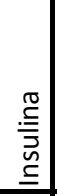 & 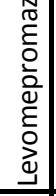 & 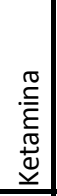 & $\begin{array}{l}\frac{\pi}{0} \\
\frac{0}{0} \\
\frac{\pi}{0} \\
\stackrel{ \pm}{0} \\
\Sigma \\
\end{array}$ & 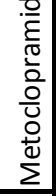 & $\begin{array}{l}\varepsilon \\
\frac{E}{0} \\
0 \\
T \\
\frac{\pi}{0} \\
\Sigma \\
\end{array}$ & \begin{tabular}{|c}
0 \\
$\stackrel{0}{5}$ \\
$\stackrel{5}{5}$ \\
$\stackrel{0}{\Sigma}$ \\
\end{tabular} & 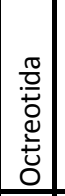 & 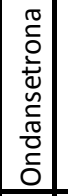 & 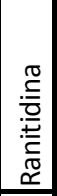 & 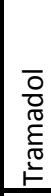 \\
\hline Ampicilina & & NT & NT & NT & NT & NT & 1 & 1 & 1 & NT & NT & I & C & NT & NT & NT & NT & NT & NT & NT & NT & NT & NT & NT & NT & NT \\
\hline Atropina & NT & & NT & NT & NT & NT & 1 & 1 & I & NT & NT & 1 & C & NT & NT & NT & NT & NT & NT & NT & NT & NT & NT & NT & NT & NT \\
\hline Cefepima & NT & NT & & NT & NT & NT & 1 & 1 & 1 & C & NT & 1 & C & C & NT & NT & C & NT & NT & C & I & C & C & C & 1 & C \\
\hline Cefotaxima & NT & NT & NT & & NT & NT & 1 & 1 & I & NT & NT & 1 & NT & NT & NT & NT & NT & NT & NT & NT & NT & NT & NT & NT & NT & NT \\
\hline Ceftriaxone & NT & NT & NT & NT & & NT & 1 & I & I & NT & NT & I & C & I & NT & NT & NT & NT & NT & C & C & C & C & I & I & NT \\
\hline Ceftazidima & NT & NT & NT & NT & NT & & 1 & 1 & I & NT & NT & 1 & NT & NT & NT & NT & NT & NT & NT & NT & NT & NT & NT & NT & NT & NT \\
\hline Cetorolaco & I & I & 1 & 1 & 1 & 1 & & I & 1 & 1 & 1 & 1 & I & I & 1 & I & I & I & I & I & 1 & 1 & I & 1 & 1 & 1 \\
\hline Dexametasona & 1 & 1 & 1 & 1 & 1 & 1 & 1 & & 1 & 1 & I & 1 & 1 & 1 & 1 & 1 & 1 & 1 & 1 & 1 & I & 1 & 1 & I & 1 & 1 \\
\hline Dipirona & I & 1 & I & 1 & 1 & 1 & 1 & 1 & & 1 & 1 & 1 & 1 & 1 & I & 1 & I & 1 & 1 & I & 1 & 1 & 1 & 1 & I & 1 \\
\hline Escopolamina & NT & NT & C & NT & NT & NT & 1 & 1 & 1 & & NT & 1 & NT & C & NT & NT & NT & NT & NT & C & C & C & C & C & NT & C \\
\hline Fentanil & NT & NT & NT & NT & NT & NT & 1 & I & I & NT & & I & NT & NT & NT & NT & NT & NT & NT & NT & NT & NT & NT & NT & NT & NT \\
\hline Fenobarbital & I & 1 & 1 & I & 1 & I & 1 & 1 & 1 & 1 & 1 & & I & I & I & I & I & I & I & I & I & 1 & I & I & I & 1 \\
\hline Furosemida & NT & NT & C & NT & C & NT & 1 & 1 & 1 & NT & NT & 1 & & I & $\mathrm{C}$ & NT & NT & NT & NT & 1 & I & 1 & NT & I & C & NT \\
\hline Haloperidol & NT & NT & C & NT & 1 & NT & 1 & 1 & 1 & C & NT & 1 & NT & & C & NT & NT & C & C & NT & C & C & NT & NT & NT & C \\
\hline Hioscina & NT & NT & NT & NT & NT & NT & 1 & 1 & I & C & NT & 1 & NT & C & & NT & NT & C & C & $\mathrm{C}$ & C & C & C & C & NT & C \\
\hline Insulina & NT & NT & NT & NT & NT & NT & 1 & 1 & I & C & NT & 1 & I & NT & NT & & C & NT & NT & C & C & C & I & NT & I & C \\
\hline Levomepromazina & NT & NT & $\mathrm{C}$ & NT & NT & NT & 1 & 1 & 1 & NT & NT & 1 & NT & $\mathrm{C}$ & NT & NT & & NT & NT & C & C & $\mathrm{C}$ & NT & NT & I & NT \\
\hline Ketamina & NT & NT & NT & NT & NT & NT & 1 & 1 & I & C & NT & 1 & NT & C & C & NT & NT & & NT & C & C & C & NT & C & NT & NT \\
\hline Metadona & NT & NT & NT & NT & NT & NT & 1 & 1 & 1 & c & NT & 1 & NT & C & C & NT & NT & NT & & C & C & 1 & NT & NT & NT & 1 \\
\hline Metoclopramida & NT & NT & C & NT & C & NT & 1 & 1 & 1 & C & NT & 1 & 1 & C & C & C & C & C & C & & C & C & C & NT & C & C \\
\hline Midazolam & NT & NT & 1 & NT & $\mathrm{C}$ & NT & 1 & 1 & 1 & $\mathrm{C}$ & NT & 1 & 1 & C & C & $\mathrm{C}$ & C & C & C & $\mathrm{C}$ & & $\mathrm{C}$ & NT & C & 1 & 1 \\
\hline Morfina & NT & NT & C & NT & C & NT & 1 & 1 & I & C & NT & 1 & I & C & C & C & C & C & 1 & C & C & & C & C & C & I \\
\hline Octreotida & NT & NT & C & NT & C & NT & 1 & 1 & I & $\mathrm{C}$ & NT & 1 & NT & NT & $\mathrm{C}$ & 1 & NT & NT & NT & $\mathrm{C}$ & NT & C & & NT & NT & NT \\
\hline Ondansetrona & NT & NT & C & NT & I & NT & 1 & 1 & I & C & NT & 1 & 1 & NT & C & NT & C & C & NT & NT & C & C & NT & & NT & NT \\
\hline Ranitidina & NT & NT & I & NT & I & I & 1 & 1 & 1 & $\mathrm{C}$ & NT & 1 & C & 1 & NT & I & I & NT & NT & C & 1 & C & NT & NT & & NT \\
\hline Tramadol & NT & NT & $C$ & NT & NT & NT & 1 & 1 & 1 & C & NT & 1 & NT & C & C & NT & NT & NT & I & C & I & 1 & NT & NT & NT & \\
\hline
\end{tabular}

Legenda: Branco = não testado (NT); Verde = compatível (C); Vermelho = incompatível (I).

\section{COMENTÁRIOS FINAIS}

A hipodermóclise é um método antigo, mas que ganha cada vez mais destaque, sendo um recurso seguro e aplicável na promoção de autonomia e conforto de pacientes idosos e/ou em cuidados paliativos, possibilitando até mesmo melhorias na adesão ao tratamento.

Os trabalhos e manuais selecionados para revisão comprovam a segurança da via subcutânea para 
pacientes idosos ou em cuidados paliativos, enfatizam a eficiência da técnica para controle e alívio de sintomas, principalmente provenientes de doenças crônicas. Evidenciou-se durante a pesquisa a divergência entre as recomendações com relação às administrações de medicamentos pela via subcutânea, por se tratar de uso off label. Para construção de nosso material, adotamos as orientações que eram mais concordantes entre os autores, porém, destaca-se a necessidade de estudar cada paciente individualmente, verificando a diluição recomendada para cada tipo de medicamento considerando o volume máximo de infusão de $3000 \mathrm{~mL}$ a cada 24 horas.

Para realizar o uso seguro e eficiente da técnica, faz-se necessária a construção de protocolos adequados às demandas cada instituição, de acordo com os medicamentos e materiais disponíveis para uso. Destacamos a importância de divulgar o conhecimento sobre novas técnicas como essa, sendo fundamental a atenção a novas atualizações no cuidado de pacientes especiais, como idosos, com doenças crônicas ou terminais para atingir seus objetivos terapêuticos.

\section{REFERÊNCIAS}

1. Campos ACV, Gonçalvez LHT. Perfil demográfico do envelhecimento nos municípios do estado do Pará, Brasil. Rev Bras Enferm. 2018;71(supl 1):636-43.

2. Miranda GMD, Mendes ACG, Silva ALA. $O$ envelhecimento populacional brasileiro: desafios e consequências sociais atuais e futuras. Rev Bras Geriatr Gerontol. 2016;19(3):507-19.

3. World Health Organization. Envelhecimento ativo: uma política de saúde. Brasília: Organização Pan-Americana da Saúde; 2005.

4. Brasil. Ministério da Saúde. Secretaria de Atenção à Saúde. Departamento de Atenção Básica. Envelhecimento e saúde da pessoa idosa. Brasília: Ministério da Saúde; 2007. https://doi.org/10.1590/s1981-77462010000100003

5. Brasil. Portaria no 2.528, de 19 de outubro de 2006. Aprova a Política Nacional de Saúde da Pessoa Idosa. Brasília: Ministério da Saúde; 2006. https://doi.org/ 10.11606/d.25.2013.tde-16072014-135015

6. Organización Mundial de la Salud. Cuidados paliativos [Internet]. Geneva: WHO; 2018 [capturado em 24 de jul. 2018]. Disponível em: http:/www.who.int/es/news-room/ fact-sheets/detail/palliative-care.

7. Capelas MLV, Coelho SPF. Pensar a organização de serviços de cuidados paliativos. Cuidados Paliativos. 2014;1(1):17-26.

8. Instituto Paliar. Cuidados paliativos [Internet]. São Paulo: Instituto Paliar; 2013 [capturado em 24 de jul. 2018]. Disponível em: http://www.paliar.com.br/cuidados- paliativos. https://doi.org/10.11606/t.5.2018.tde27092018-092046

9. Zironde ES, Marzenini NL, Soler VM. Hipodermóclise: redescoberta da via subcutânea no tratamento de indivíduos vulneráveis. CuidArte Enferm. 2014;8(1):55-61.

10. Ferreira KASL, Santos AC. Hipodermóclise e administração de medicamentos por via subcutânea: uma técnica do passado com futuro. Prática Hospitalar. 2009;9(65):109-14.

11. Pontalti G, Rodrigues ESA, Firmino F, Fabris M, Stein MR, Longaray VK. Via subcutânea: segunda opção em cuidados paliativos. Rev HCPA. 2012;32 (2):199-207.

12. Conselho Federal de Enfermagem do Distrito Federal. Parecer Técnico do Coren-DF. 006/2014. A manipulação/ inserção do método hipodermóclise em domicílio. Brasília: Coren-DF; 2015. https://doi.org/10.20873/abef.2595-0096. v1n2p55.2018

13. Nunes PMSA, Souza RCS. Adverse effects of hypodermoclysis in adult patients: an integrative review. REME: Rev Min Enferm. 2016;20(951):1-5.

14. Pontalti G, Riboldi CO, Gioda RS, Echer IC, Franzoi MA, Wegner W. Benefícios da hipodermóclise na clínica paliativa de pacientes com câncer: relato de caso. Rev Bras Cancerol. 2016;62(3):247-52. https://doi.org/ 10.32635/2176-9745.rbc.2016v62n3.222

15. Azevedo DL. O uso da via subcutânea em geriatria e cuidados paliativos. 2. ed. Rio de Janeiro: SBGG; 2016.

16. Vidal FKG, Oselame GB, Neves EB, Oliveira EM. Hipodermóclise: revisão sistemática da literatura. Rev Atenção Saúde. 2015;13(45):61-9. https://doi. org/10.13037/ras.vol13n45.2953

17. Word Health Organization. WHO definition of palliative care [Internet]. Geneva: WHO; 2019 [capturado em 24 de jul. 2018]. Disponível em: http://www.who.int/cancer/ palliative/definition/en/.

18. Dalacorte RR, Rigo JC, Schneider RH, Schwanke $\mathrm{CHA}$, organizadores. Cuidados paliativos em geriatria e gerontologia. São Paulo: Atheneu; 2012.

19. Garcia JBS, Rodrigues RF, Lima SF. A estruturação de um serviço de cuidados paliativos no Brasil: relato de experiência. Rev Bras Anestesiol. 2014;64(4):286-91. https://doi.org/10.1016/j.bjan.2013.06.007

20. Godinho NC, Silveira LVA. Manual de hipodermóclise [Internet]. Botucatu: Hospital de Clínicas da Faculdade de Medicina de Botucatu; 2017 [capturado em 20 mar. 2018]. Disponível em: http://www.hcfmb. unesp.br/wp-content/uploads/2017/12/Manual-deHipoderm\%C3\%B3clise-HCFMB.pdf. https://doi. org/10.31692/2526-7701.iicointerpdvagro.2017.00100

21. Bruno VG. Hipodermóclise: revisão da literatura para auxiliar a prática clínica. Einstein. 2015;13(1):122-28.

22. Azevedo EF, Barbosa LA, Cassiani SHB. Administração de antibióticos por via subcutânea: uma revisão integrativa da literatura. Acta Paul Enferm. 2012;25(5):817-22. https://doi.org/10.1590/s0103-21002012000500026 
23. Gomes NS, Silva AMB, Zago LB, Silva ECL, Barrichello E. Conhecimentos e práticas da enfermagem na administração de fluidos por via subcutânea. Rev Bras Enferm. 2017;70(5):1155-64.

24. Brasil. Ministério da Saúde. Instituto Nacional do Câncer. Terapia subcutânea no câncer avançado. Rio de Janeiro: INCA 2009. https://doi.org/10.5152/ejbh.2019.4414

25. Cunha MG, Cunha ALG, Machado CA. Hypodermis and subcutaneous adipose tissue: two different structures. Surg Cosmet Dermatol. 2014;6(4):355-9.

26. Justino ET, Tuoto FS, Kalinke LP, Mantovani MF. Hipodermóclise em pacientes oncológicos sob cuidados paliativos. Cogitare Enferm. 2013;18(1):84-9. https://doi.org/10.5380/ce.v18i1.31307

27. Carvalho RT, Parsons HA. Manual de cuidados paliativos ANCP [Internet]. 2. ed. Rio de Janeiro: ANCP; 2012 [capturado em 20 mar. 2018]. Disponível em: http:// biblioteca.cofen.gov.br/wp-content/uploads/2017/05/ Manual-de-cuidados-paliativos-ANCP.pdf. https://doi. org/10.11606/t.5.2018.tde-27092018-092046

28. O'Hanlon S, Sheahan P, McEneaney R. Severe hemorrhage from a hypodermoclysis sit. Am J Hosp Palliat Med. 2009;26(2):135-6. https://doi.org/10.1177/ 1049909108330033

29. Braz CL, Pereira RCC, Costa JM. Administração de medicamentos por hipodermóclise: uma revisão da literatura. Rev Bras Farm Hosp Serv Saúde São Paulo. 2015;6(1):6-12.

30. Vidal M, Hui D, Williams J, Bruera E. A prospective study of hypodermoclysis performed by caregivers in home setting. J Pain Symptom Manage. 2016;52(4):570-4.e9. https://doi.org/10.1016/j.jpainsymman.2016.04.009

31. Hospital Israelita Albert Einstein. Manual Farmacêutico [Internet]. São Paulo; 2017 [capturado em 20 mar. 2018]. Disponível em: https://aplicacoes.einstein.br/ manualfarmaceutico/Paginas/Termos.aspx?filtro $=$ ta belas\&itemID $=167$. https://doi.org/10.1590/s1679. 45082012000200001

\section{AUTHORS:}

CAMILA FIgueIRÓ VASCONCELLOS

Farmacêutica formada pela Pontifícia Universidade Católica do Rio Grande do Sul, especialista em farmácia hospitalar e clínica pelo Instituto de Educação e Pesquisa do Hospital Moinhos de Vento, residente em apoio diagnóstico e terapêutico no Hospital São Lucas da PUCRS. Responsável pela construção do trabalho.

E-mail: camila.vasconcellos@acad.pucrs.br

Orcid: https://orcid.org/0000-0003-4316-2205

DENISE MILÃO

Farmacêutica formada pela Universidade Federal do Rio Grande do Sul, mestre em ciências farmacêuticas pela Universidade do Rio Grande do Sul, professora da Escola de Ciências da Saúde da Pontifícia Universidade Católica do Rio Grande do Sul.

Responsável pela revisão do trabalho.

E-mail:dmilao@pucrs.br

Orcid: https://orcid.org/0000-0003-0826-103X 